\title{
Design of Translucent Optical Networks: Partitioning and Restoration
}

\author{
Ezhan Karasan* \\ Department of Electrical and Electronics Engineering, Bilkent University, TR-06800 Ankara, Turkey \\ E-mail:ezhan@ee.bilkent.edu.tr \\ Mustafa Arisoylu \\ Department of Electrical and Computer Engineering, University of California at San Diego, La Jolla, CA 92093-0407, USA \\ E-mail: marisoyl@ucsd.edu
}

Received July 24, 2003; Accepted November 7, 2003

\begin{abstract}
We discuss the problem of designing translucent optical networks composed of restorable, transparent subnetworks interconnected via transponders. We develop an integer linear programming (ILP) formulation for partitioning an optical network topology into subnetworks, where the subnetworks are determined subject to the constraints that each subnetwork satisfies size limitations, and it is two-connected. A greedy heuristic partitioning algorithm is proposed for planar network topologies.

We use section restoration for translucent networks where failed connections are rerouted within the subnetwork which contains the failed link. The network design problem of determining working and restoration capacities with section restoration is formulated as an ILP problem. Numerical results show that fiber costs with section restoration are close to those with path restoration for mesh topologies used in this study. It is also shown that the number of transponders with the translucent network architecture is substantially reduced compared to opaque networks.
\end{abstract}

Keywords: translucent optical networks, subnetwork partitioning, section restoration

\section{Introduction}

Multiwavelength optical networks are currently widely deployed in long-distance core networks. There are several architectures suitable for optical networks, each involving complex combinations of optical and electronic devices. All these architectures have the optical fiber as the transmission medium and contain some form of optical cross-connects (OXC) interconnecting these fibers.

In transparent or all-optical networks architecture, a connection goes through the network over a completely optical path $[1,2]$. This path consists of pointto-point optical links interconnected by all-optical nodes. Wavelength selective cross-connects (WSXC) can switch an incoming connection on some wavelength onto the same wavelength in any of its outgoing fibers. In transparent networks utilizing WSXCs, connections must satisfy the wavelength continuity constraint, that is, a connection must remain on the same wavelength on all links along its path. On the other hand, wavelength interchanging cross-connects (WIXC) employ optical wavelength conversion in order to switch an incoming connection onto any wavelength in any outgoing fiber. As such, they enable more efficient packing of wavelengths onto the fibers by eliminating wavelength conflicts. Optical wavelength converter technologies have relatively high costs, making WIXCs significantly more expensive compared to WSXCs [3]. Performance of all-optical networks with and without wavelength converters have been studied extensively in the literature [4-6].

A second type of optical networks is the opaque network architecture [7]. In opaque networks, a connection passes through optical/electronic/optical converters, called transponders, at each node. Opaque networks may utilize optical or electronic form of switching at intermediate nodes. Optical networks that are deployed today are typically opaque, and they use electronic cross-connects (EXC) [8].

One of the main advantages of all-optical networks is transparency. There are various levels of 
transparency delivered by all-optical networks such as bit rate, protocol, modulation format and analog/ digital transparencies. Transparency enables easier deployment of new technologies. However, transparency also brings a cost due to accumulated impairments in optical transmission and switching systems requiring regeneration of optical signals.

Because of the limitations of building nationalscale multi-vendor all-optical networks and the high cost of opaque networks, a new optical network architecture is recently considered [8-11]. In this formation, there are islands of transparency, that is, all-optical subnetworks, each having a limited geographic size. These subnetworks are interconnected to each other via non-transparent transponders forming a translucent optical network. Translucent networks have several advantages over previously known architectures: easier multi-vendor interoperability, reduced transponder costs, simpler network management, scalable network architecture and faster restoration times. Another implementation of a translucent network is based on sparse placement of opaque nodes where wavelength conversion and regeneration are possible $[9,12]$.

In this paper, we discuss two interrelated problems in the design of translucent networks. First, we describe how a given topology can be partitioned into transparent subnetworks such that each subnetwork is restorable against all possible single-link failures. We formulate this problem as an ILP problem where the network is partitioned into the smallest number of subnetworks subject to the constraints that each subnetwork satisfies some size constraints, and it is two-connected. The network partitioning problem is solved by only using the topology of the network independent of the traffic, since partitioning must be done at the initial network deployment phase. A greedy heuristic algorithm for the partitioning problem is also proposed for planar network topologies.

After the partitioning problem is solved, the network design problem of determining working and restoration capacities using the proposed section restoration in translucent networks is studied. The resulting optimization problem is formulated as an ILP problem. Although network partitioning and capacity design problems are coupled to each other, we consider them separately because of the large computational complexity of the joint problem. Unlike the partitioning problem, the solution of the capacity design problem depends on the offered traffic since network resources can be dynamically provisioned with response to changes in the traffic demand.

After presenting the section restoration technique for translucent network architecture in Section 2, we discuss the network partitioning problem in Section 3. In Section 4, the network design problem of computing working and restoration capacities is considered. Conclusions of the paper are presented in Section 5.

\section{Section Restoration in Translucent Optical Networks}

As optical networking becomes more commonplace in core transport networks, optical switching technologies will be introduced in more network nodes. When the number of optical nodes in the network is large, the design and management of the network become more complicated. Translucent networks partition a network topology into multiple subnetworks. Each of these subnetworks can be designed and managed separately, simplifying these problems substantially. This results in a more scalable network architecture: as the number of nodes in the network increases the number of subnetworks will increase, but not the sizes of these subnetworks.

Translucent networks also provide simplified operation, administration, and management (OAM). One of the most important OAM functionalities is restoration. In translucent networks, the restoration function can be managed by multiple restoration managers (RM), one for each subnetwork. Each RM is held accountable for restoring failures within its own subnetwork. Each RM keeps a separate database containing all information for the connections routed through its own subnetwork. In this paper, we consider restoration in the optical layer in response to single-link failures. When a link fails, the corresponding RM reroutes all failed connections within its subnetwork without communicating with other RMs. Therefore, no interworking is necessary between RMs for restoring connections after a failure. This restoration technique is called section restoration. Rerouting techniques similar to the section restoration where only a portion of the working path is rerouted have been proposed in the literature $[13,14]$. 
In section restoration, when a link within subnetwork $S_{i}$ fails, the transponders located at the boundaries of $S_{i}$ detect the failure of each affected connection without requiring any optical monitoring. If a failed connection has its source and/or destination nodes in $S_{i}$, then the failure is detected by the transceivers at the source/destination nodes. Failure information is forwarded to the RM for $S_{i}$ which handles the restoration of all failed connections. Each failed connection is rerouted between its entry and exit points of subnetwork $S_{i}$ such that the restoration path is link-disjoint from the working path within $S_{i}$. Section restoration is a failure-independent scheme since fault localization within the subnetwork is not necessary.

Path restoration, where failed connections are rerouted between the source and destination nodes, have been studied extensively in the literature. Routing and capacity planning for path restoration is discussed in Alanyali and Ayanoglu [15], Mohan and Murthy [16], Iraschko and Grover [17], and Doshi et al. [18]. Section restoration is a special form of path rerouting where paths are restricted to subnetworks.

Section restoration is a quasi-distributed technique where after a link failure a single RM reroutes the affected traffic independent of other RMs. Since the size of each subnetwork is relatively small, propagation of failure information within the subnetwork to the corresponding RM and reconfiguration of OXCs can be accomplished in shorter time with section restoration compared to path restoration where failed connections are rerouted between the source and destination nodes. In section restoration, the restoration path may use a different wavelength than the working path within the failed subnetwork since transponders and tunable laser/receivers can perform wavelength conversion.

With section restoration, only the nodes at the boundaries of the subnetworks need to carry restoration functions. Interior nodes are passive while the restoration function is carried out except that they need to report failures of adjacent links to RM.

In this paper, we consider preplanned restoration where working and restoration link capacities are computed for static traffic demand such that all connections can be restored in the case of any singlelink failure. Section restoration is a shared restoration technique where restoration capacities are shared among link-disjoint working paths.
In the next section, we discuss the problem of partitioning the network into subnetworks subject to size and connectivity constraints.

\section{Network Partitioning}

Subnetworks in a translucent optical network may need to satisfy several properties. First, each subnetwork should be two-connected, that is, there should exist at least two link-disjoint paths between any two nodes in the same subnetwork. This is necessary for guaranteeing full restoration against any single-link failure. We assume that the network topology corresponds to a graph that can be partitioned into two-connected subnetworks. A trivial necessary condition for this is the two-connectivity of the original network topology.

Next, the size of the subnetworks may need to be below some given thresholds. If each subnetwork contains a small number of links and nodes, RMs deal with smaller databases that leads to a more scalable architecture with shorter restoration times. In this paper, the lengths of working and restoration paths between any two nodes in the same subnetwork can be at most $R_{\max }$. We also restrict the number of nodes in a subnetwork to $N_{\max }$ and the number of links to $L_{\max }$ so that computational complexity of the network design problem for each subnetwork is bounded. With smallsized subnetworks, restoration capacities are used inefficiently since section restoration in this case resembles link rerouting. Therefore, the number of subnetworks is minimized as an objective.

For restoration purposes, each link should belong to only one subnetwork, that is, when a link fails, RM of the related subnetwork will be responsible for restoration of all failed connections. However, for partitioning purposes, a link may be shared between two or more subnetworks in order to satisfy twoconnectivity for each subnetwork. This can be described by introducing a two-layer structure. In the restoration layer, each link belongs to a single subnetwork, that is, when a link fails the corresponding RM handles the failure. On the other hand, in the overlapped subnetworks layer some links may be shared between two or more subnetworks so that each subnetwork is two-connected. The links shared in the overlapped subnetworks layer may be carrying restored traffic resulting from a failure in one of the 
sharing subnetworks. Since we only consider singlelink failures, a shared link can be used by rerouted traffic directed by at most one RM after any failure.

Based on the above constraints, two solutions for network partitioning problem are presented below: The first one based on an ILP formulation and the other one based on a greedy heuristic algorithm.

\subsection{Partitioning with ILP Formulation}

The ILP formulation for the network partitioning problem consists of two parts. First, we compute all link-disjoint paths between each node pair. In the second part, the network is divided into twoconnected subnetworks using the link-disjoint paths computed in the first part.

We first find the maximum number of link-disjoint paths for each node pair such that all paths have lengths not exceeding $R_{\max }$. We compute these paths in two stages. First, the unconstrained max-flow problem is solved, that is, for each node pair the maximum number of flows that can be carried between these nodes is found such that the total length of all used links is minimized [19]. At the second stage, link-disjoint paths passing through the links with positive flows in the first stage are found subject to the constraint that all paths have lengths not exceeding $R_{\max }$. These steps are repeated subsequently for all node pairs.

Suppose the network topology is represented by an undirected graph $G=(V, E)$, where $V$ is the node set and $E$ is the set of links. Let $P^{m n}$ denote the set of constrained link-disjoint paths between nodes $m$ and $n$ found at the end of the first part, and $\left|P^{m n}\right|$ corresponds to the number of such paths. Let $P_{k}^{m n}$ denote $k$-th shortest path in $P^{m n}$, and let $\left|P_{k}^{m n}\right|$ denote the number of links on path $P_{k}^{m n}$. Let the indicator function $\delta_{l n}$ describe the node-link adjacency defined as $\delta_{l n}=1$ if link $l$ is adjacent to node $n$. The set of possible subnetworks is denoted by $S$.

We define the following binary decision variables: $Y_{n s}=1$, if node $n$ is in subnetwork $s$, and $U_{l s}=1$, if link $l$ is in subnetwork $s$. Next, we define the following auxiliary variables: $Z_{m n s}=1$, if $Y_{m s}=Y_{n s}=1$, that is, both nodes $m$ and $n$ are in subnetwork $s, W_{k s}^{m n}=1$, if $P_{k}^{m n}$ lies completely inside subnetwork $s$, and $T_{s}=1$, if at least a link is included in subnetwork $s$.

The ILP formulation for the network partitioning problem is given by:
Objective: Minimize $\sum_{s \in S} T_{s}+\varepsilon \sum_{s \in S} \sum_{l \in E} U_{l s}$ Subject to:

$$
\begin{aligned}
& \sum_{s \in S} U_{l s} \geq 1, \quad \forall l \in E, \\
& Y_{n s} \geq U_{l s} \delta_{l n}, \quad \forall n \in V, \forall s \in S, \forall l \in E, \\
& Y_{n s} \leq \sum_{l \in E} U_{l s} \delta_{l n}, \quad \forall n \in V, \forall s \in S, \\
& \sum_{l \in E} U_{l s} \leq L_{\max }, \quad \forall s \in S, \\
& \sum_{n \in V} Y_{n s} \leq N_{\max }, \quad \forall s \in S, \\
& Z_{m n s} \leq Y_{m s}, \quad \forall(m, n) \in V \times V, \forall s \in S, \\
& Z_{m n s} \leq Y_{n s}, \quad \forall(m, n) \in V \times V, \forall s \in S, \\
& Z_{m n s} \geq Y_{n s}+Y_{m s}-1 \text {, } \\
& \forall(m, n) \in V \times V, \forall s \in S, \\
& W_{k s}^{m n} \leq U_{l s}, \quad \forall l \in P_{k}^{m n}, \forall s \in S, \forall k \in\left[1,\left|P^{m n}\right|\right], \\
& \forall(m, n) \in V \times V, \\
& W_{k s}^{m n} \geq \sum_{l \in P_{k}^{m n}} U_{l s}-\left|P_{k}^{m n}\right|+1, \\
& \forall s \in S, \forall k \in\left[1,\left|P^{m n}\right|\right], \forall(m, n) \in V \times V, \\
& \sum_{k=1}^{\left|P^{m n}\right|} W_{k s}^{m n} \geq 2 Z_{m n s}, \quad \forall(m, n) \in V \times V, \forall s \in S, \\
& U_{l s} \leq T_{s}, \quad \forall l \in E, \\
& Y_{n s}, U_{l s}, Z_{m n s}, W_{k s}^{m n} \in\{0,1\} \text {. }
\end{aligned}
$$

The objective of the formulation is to minimize not only the total number of subnetworks used, but also the total number of shared links. Both objectives are embedded into one objective function by multiplying the latter one with a small constant $\varepsilon$ such that the minimization of the number of subnetworks has the highest priority. For example, $\varepsilon$ can be chosen as $\varepsilon=1 /(|V||E|)$, where $|V|$ and $|E|$ correspond to the number of nodes and edges, respectively. By reducing the number of shared links, we make the design of individual subnetworks more independent from each other.

Constraint (1) expresses that each link must belong to at least one subnetwork. Constraints (2-3) state that if one adjacent link of node $n$ belongs to subnetwork $s$, then node $n$ is also part of subnetwork $s$. Constraints (4-5) guarantee that each subnetwork satisfies node and link size constraints. Constraints (6-8) imply that if both nodes $m$ and $n$ are in subnetwork $s$, then the variable $Z_{m n s}=1$, otherwise $Z_{m n s}=0$. Constraints 
$(9-10)$ indicate that in order to have $W_{k s}^{m n}=1$, all the links on path $P_{k}^{m n}$ should reside in subnetwork $s$, otherwise $W_{k s}^{m n}=0$. Constraint (11) provides the twoconnectivity requirement for each subnetwork. If nodes $m$ and $n$ are in subnetwork $s$, that is, $Z_{m n s}=1$, then there should be at least two link-disjoint paths between nodes $m$ and $n$ lying completely inside subnetwork $s$. If there are no such paths, $Z_{m n s}=0$. Finally, constraint (12) ensures the existence of a subnetwork if at least one link belongs to that subnetwork.

\subsection{Greedy Heuristic Partitioning Algorithm}

Due to the high computational complexity of solving the ILP problem, it may not be possible to obtain the solution for large networks within a run-time limit. Therefore, we propose a heuristic algorithm for planar network topologies. A planar graph can be drawn in the plane such that its edges intersect only at their common end-vertices [20]. For a planar graph, the regions bounded by the edges are called faces. The links surrounding a face constitute a simple cycle which is two-connected. Two faces are called adjacent if they share at least one edge. The number of faces of a connected planar graph $G=(V, E)$ is given by the Euler formula

$$
|F|=|E|-|V|+2
$$

where $|E|$ is the number of edges and $|V|$ is the number of nodes in $G$.

The heuristic algorithm can be described in two parts. In the first part, we find all $|F|$ faces of the planar graph corresponding to the network topology. There are several algorithms for finding the faces in a planar graph [21]. After faces are found, we combine adjacent faces to form the subnetworks which satisfy size constraints. The two-connectivity constraint is met since individual faces and combinations of adjacent faces are two-connected.

Before stating the algorithm used in the heuristic algorithm, we make the following definition.

Definition: For a given graph $G$, let $P_{1}, P_{2}, \ldots, P_{K}$ be the set of all link-disjoint paths between nodes $m$ and $n$ that are computed as described in Section 3. Let $\left|P_{i}\right|$ denote the length of $P_{i}$. The dilation of $m$ and $n$ in topology $G$ is defined as

$$
\operatorname{dil}(m, n \mid G)=\frac{\max _{i=1,2, \ldots, K}\left|P_{i}\right|}{\min _{i=1,2, \ldots, K}\left|P_{i}\right|} .
$$

The face combining algorithm starts with a cluster which is initially a single face, and then begins to enlarge the cluster by combining it with adjacent faces. At each step, a candidate cluster is formed by joining the current cluster with one of its adjacent faces such that the newly formed cluster satisfies all size constraints. For all node pairs in each candidate cluster, all link-disjoint paths are calculated, and the average dilation is computed for each candidate cluster where the average is taken over all node pairs in the cluster. Among all candidate clusters, the one with the minimum average dilation is chosen, and the corresponding adjacent face is added to the current cluster to form the new cluster.

We continue enlarging the current cluster until no candidate cluster can be found. Then a new cluster is initiated with a face which is not included in any other cluster, and the same process is repeated until all faces are included in some cluster. The clusters obtained at the termination of the algorithm form the subnetworks.

The algorithm is described as follows:

1. Initiate a new cluster with an arbitrarily selected face which is not included in any other cluster. If no such face exists, stop.

2. For the current cluster find the candidate cluster that has the minimum average dilation. If no candidate cluster exists, go to Step 1.

3. Set current cluster to the candidate cluster found in Step 2, and go to Step 2.

\subsection{Numerical Results}

ILP formulation and the greedy heuristic algorithm are applied to two different network topologies. The 32-node topology is an approximation of a carrier's core network where nodes correspond to major US cities [15]. The 23-node mesh network shown in Fig. 1 is the right-half of the 32-node topology. The lengths of the links in these networks are the actual distances between major cities corresponding to nodes.

For the 23-node network various kinds of size constraints were used in the partitioning process. We have used the same constraints for both the ILP formulation and the heuristic algorithm. The ILP formulation was implemented on CPLEX 7.1. For $R_{\max }=2500 \mathrm{~km}, N_{\max }=12$ and $L_{\max }=15$, partition- 


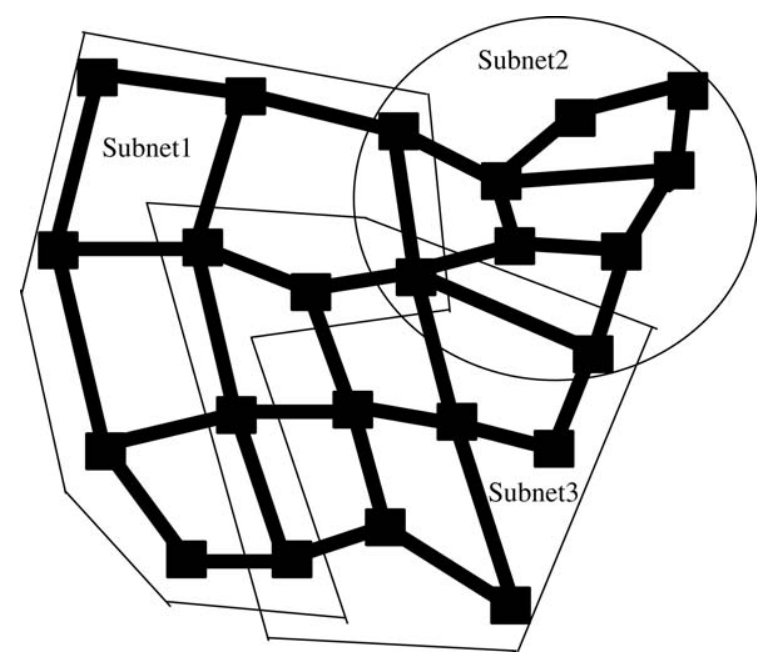

Fig. 1. Network partitioning for 23-node network (ILP solution) with $R_{\max }=2500 \mathrm{~km}, L_{\max }=15, N_{\max }=12, \quad$ and with $R_{\max }=3000 \mathrm{~km}, L_{\max }=15, N_{\max }=12$.

ings with both methods for the 23-node network are shown in Figs 1 and 2(a). The ILP formulation generated three subnetworks whereas the heuristic algorithm produced four subnetworks. When we increase $R_{\max }$ from $2500 \mathrm{~km}$ to $3000 \mathrm{~km}$ and leave the other parameters the same as in the previous example, the ILP formulation produces again the same three subnetworks, while the heuristic algorithm generates four subnetworks as shown in Fig. 2(b).

On the other hand, with the parameters $R_{\max }=2500 \mathrm{~km}, N_{\max }=16$, and $L_{\max }=24$, the partitionings produced by the two methods look similar as shown in Figs 3(a) and 4(a): both methods produce three subnetworks. When we increase $R_{\max }$ to
$3000 \mathrm{~km}$, a slight difference in partitioning with the heuristic algorithm occurs as can be seen in Fig. 4(b), where the number of the subnetworks does not change, however their sizes are altered. With the same parameters, the output of the ILP formulation changes significantly as shown in Fig. 3(b) where two subnetworks are generated with sizes of 11 and 15 nodes leading to a significant reduction in the objective cost, that is, both the number of subnetworks and the number of shared links are reduced.

For the 32-node network, two sets of size constraints were used: $R_{\max }=3000 \mathrm{~km}, N_{\max }=16$, and $R_{\max }=3000 \mathrm{~km}, L_{\max }=24$ and $N_{\max }=12$ and $L_{\max }=15$. The ILP formulation was not able to generate a reasonable solution within a runtime of 36 hours. The greedy heuristic algorithm produced a partitioning with five subnetworks shown in Fig. 5(a) for $N_{\max }=16$ and $L_{\max }=24$ case. With the parameters $N_{\max }=12$ and $L_{\max }=15$, the algorithm generated six subnetworks as shown in Fig. 5(b). We observe from the solutions of the heuristic algorithm that it is inclined to build a very large subnetwork close to size constraints. This can be explained by the greedy nature of the heuristic, that is, it tries to enlarge the current subnetwork as much as possible without considering the rest of the network topology.

\section{Design of Working and Restoration Capacities}

In the previous section, we have partitioned the network where some links may be shared between subnetworks in for the overlapped subnetworks layer. In this section, we first determine how these shared

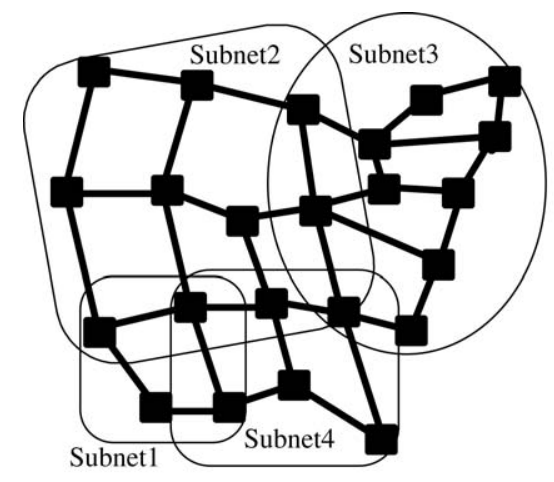

(a)

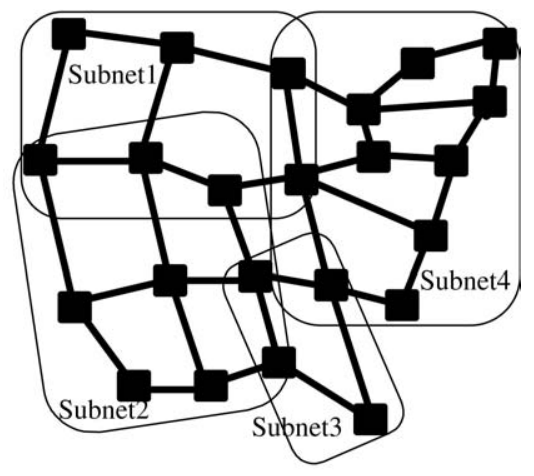

(b)

Fig. 2. Network partitioning for 23-node network (Heuristic solution) with $L_{\max }=15, N_{\max }=12$, (a) $R_{\max }=2500 \mathrm{~km}$, (b) $R_{\max }=3000 \mathrm{~km}$. 


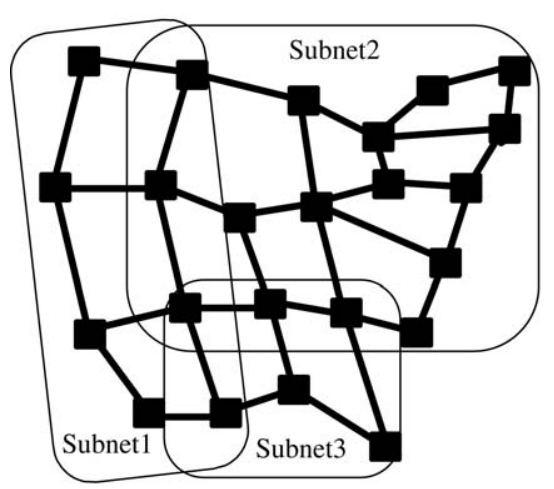

(a)

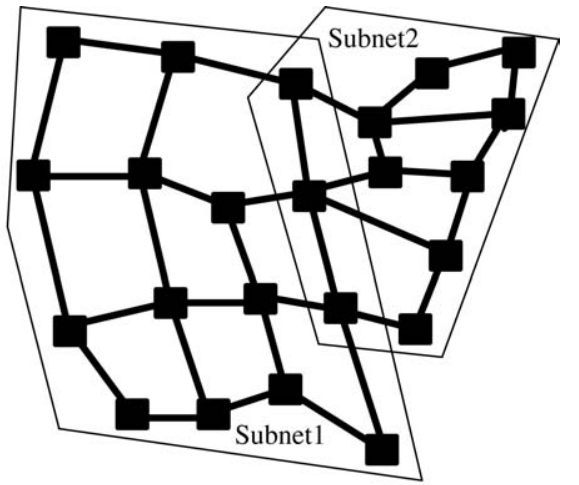

(b)

Fig. 3. Network partitioning for 23-node network (ILP solution) with $L_{\max }=24, N_{\max }=16$, (a) $R_{\max }=2500 \mathrm{~km}$, (b) $R_{\max }=3000 \mathrm{~km}$.

links are partitioned in the restoration layer, that is, for each shared link the RM in charge of the recovery of paths passing through that link is determined. We call this problem the "Link Partitioning Problem". We will then analyze the capacity planning problem for the section restoration in translucent networks and present numerical results comparing fiber costs obtained with section restoration and path restoration.

\subsection{Link Partitioning in Restoration Layer}

A heuristic algorithm for the link partitioning problem is presented. Let $\Sigma_{l}$ denote the set of subnetworks sharing link $l$. For each subnetwork $S \in \Sigma_{l}$, all linkdisjoint paths between all node pairs in $S$ are computed. Let $\tilde{P}_{l}^{S}$ denote the set of node pairs $(m, n), m, n \in S$ such that each such node pair has one of its link-disjoint paths passing through $l$. For each $(m, n) \in \tilde{P}_{l}^{S}$, we compute the average alternate path length, $\pi_{l}^{S}(m, n)$, over all link-disjoint paths except the one passing through $l$. We then compute the average alternate path length for subnetwork $S, \Pi_{l}^{S}$, which is given by

$$
\Pi_{l}^{S}=\frac{1}{\left|\tilde{P}_{l}^{S}\right|} \sum_{(m, n) \in \tilde{P}_{l}^{S}} \pi_{l}^{S}(m, n),
$$

where $\left|\tilde{P}_{l}^{S}\right|$ is the cardinality of set $\tilde{P}_{l}^{S}$. Link $l$ is then assigned in the restoration layer to subnetwork $S^{*}$ such that

$$
S^{*}=\arg \min _{S \in \Sigma_{l}} \Pi_{l}^{S}
$$

This algorithm chooses the subnetwork which has the minimum average alternate path length. The idea behind this heuristic is that alternate paths are restoration path candidates in case of failure of link $l$. The algorithm assigns $l$ to the subnetwork which has the minimum average length of restoration path candidates.

After forming the restoration layer, we proceed to the network design phase where working and restoration paths and wavelengths are selected, and appropriate link capacities are determined.

\subsection{Routing and Capacity Planning with Section Restoration}

We discuss the network design problem of determining working and restoration capacities so that the network traffic is fully restorable against all singlelink failures. We assume that the network is partitioned into all-optical WSXC-based subnetworks. Although wavelength conversion is not possible inside subnetworks, it can be performed at the boundaries of the subnetworks. We further assume that the traffic demand is static, and there is one unit wavelength of traffic for each demand. The case where there are multiple units of traffic between some node pairs can also be modeled in this framework by placing multiple one-unit traffic demands between those nodes. We also assume that the working and restoration fibers are separate. We consider two different design approaches: 


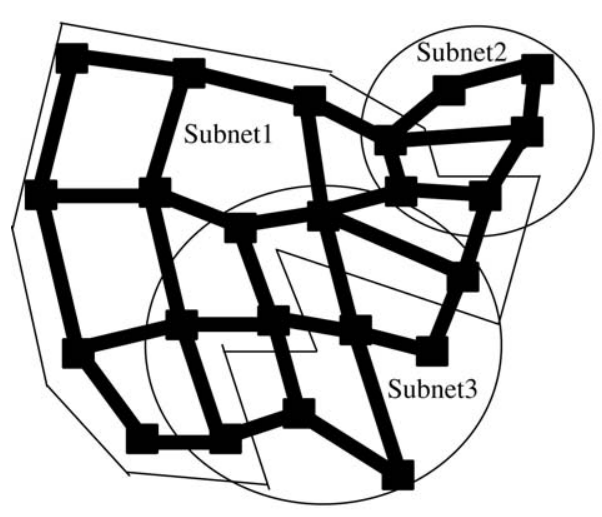

(a)

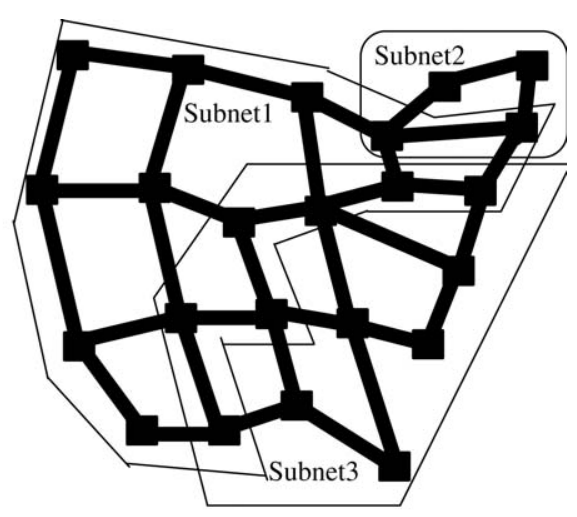

(b)

Fig. 4. Network partitioning for 23-node network (Heuristic solution) with $L_{\max }=24, N_{\max }=16$, (a) $R_{\max }=2500 \mathrm{~km}$, (b) $R_{\max }=3000 \mathrm{~km}$.

- Joint design: All link capacities are optimized for the entire network, that is, all subnetworks are jointly designed for both working and restoration capacities.

- Separate design: First, working paths and capacities are designed for the entire network independent of partitioning of the network and restoration capacities. Then, for each subnetwork restoration capacities are computed considering all single-link failures. This design is carried out for each subnetwork independent of other subnetworks. The restoration capacity for link $l$ is determined by taking the maximum capacity assignment made on link $l$ among all subnetworks sharing $l$.

The optimum solution of the joint design has lower cost than the separate design. However, the computa-

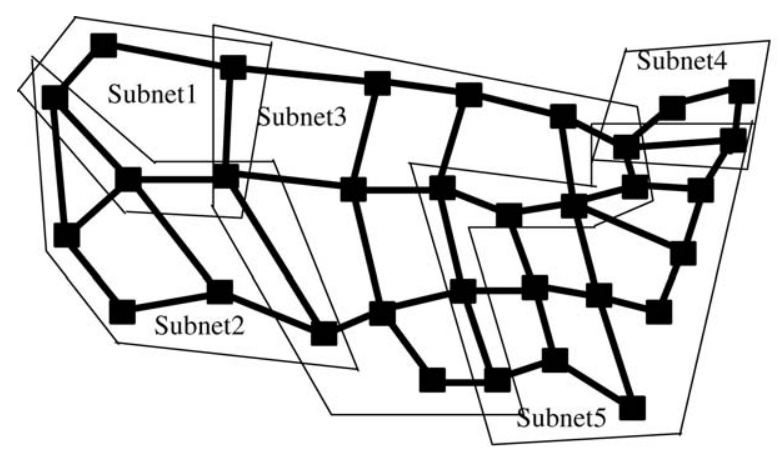

(a) tional complexity of obtaining the solution for joint design is much larger than the separate design. As the number of nodes in the network increases, computing the optimal solution for the joint design becomes extremely difficult since computational complexity is growing exponentially. As the number of nodes increases, number of subnetworks also increases, but subnetwork sizes remain limited since they are determined by the size constraints. Hence, the separate design approach is more scalable.

\subsubsection{Joint Design}

An ILP formulation is presented for planning the network by jointly considering the working and restoration capacities. The formulation presented here is a path based formulation, so the working and restoration paths are predetermined. We first find the set of $K$-shortest paths, not necessarily link-disjoint,

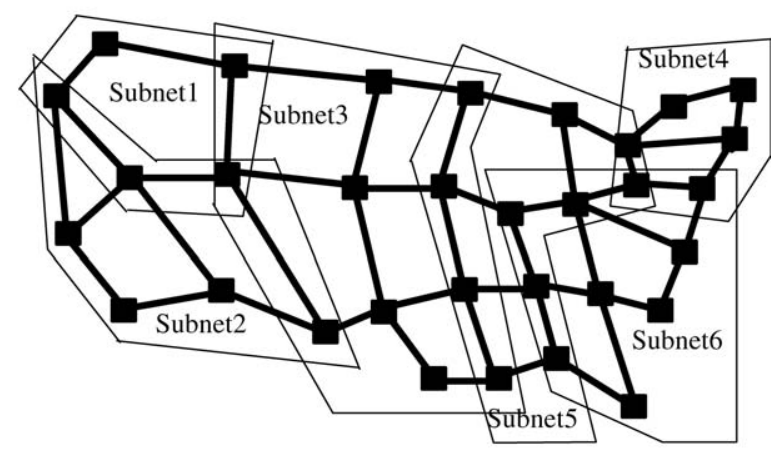

(b)

Fig. 5. Network partitioning for 32-node network (Heuristic solution) with, (a) $L_{\max }=24, N_{\max }=16, R_{\max }=3000 \mathrm{~km}, \quad$ (b) $L_{\max }=15, N_{\max }=12, R_{\max }=3000 \mathrm{~km}$. 
for all demand pairs. These paths are the candidates for working paths. Each path is then divided into sections such that all links on each section are assigned to the same subnetwork in the restoration layer. We perform this operation in such a way that all sections are maximal, that is, no two sections can be combined. For the failure of each link, $K^{\prime}$ restoration path candidates for each failed connection are computed between the entry and exit nodes of the subnetwork which contains the failed link. Inside this subnetwork, all candidate restoration paths are linkdisjoint from the failed path. However, restoration paths have exactly the same sections as the failed path except for the failed section. Furthermore, restoration capacities are computed such that they can be shared between link-disjoint working paths.

Let $D$ denote the set of node pairs for which a traffic demand exists. Let $P^{m n}$ denote the set of $K$-shortest paths for demand pair $(m, n) \in D$, and $P_{k}^{m n}$ corresponds to the $k$-th shortest path in $P^{m n}$. Each path is divided into its sections given by

$$
P_{k}^{m n}=\bigcup_{i=1}^{t_{k}^{m n}} P_{k i}^{m n}
$$

where $P_{k i}^{m n}$ is the $i$-th section of $P_{k}^{m n}$, and $t_{k}^{m n}$ is the number of sections on $P_{k}^{m n}$. For each $l \in P_{k}^{m n}, R_{l k j}^{m n}$ denotes the $j$-th restoration path for $P_{k}^{m n}$ in case of failure of link 1 . Let $d_{l}$ denote the length of link $l$, and the number of wavelengths per fiber is denoted by $W$.

We define the following indicator functions: $\beta_{l k}^{m n}=1$, if $P_{k}^{m n}$ passes through link $l, \gamma_{\bar{l} k i l}^{m n}=1$, if $R_{\overline{l k j}}^{m n}$ passes through link $l$, and $\xi_{l k i}^{m n}=1$, if $P_{k i}^{m n}$ passes through link $l$. Binary decision variables are given by $Z_{k}^{m n}=1$, if $P_{k}^{m n}$ is chosen as the working path for demand pair $(m, n), X_{k i w}^{m n}=1$, if wavelength $w$ is assigned for working path on $P_{k i}^{m n}$, and $Y_{l k j w}^{m n}=1$, if $R_{l k j}^{m n}$ is assigned as the restoration path using wavelength $w$ when link $l$ fails. Decision variables $W C_{l}$ and $R C_{l}$ correspond to the number of fibers on link $l$ used by working and restoration paths, respectively.

The optimum joint network design problem is given by

Objective: minimize $\sum_{l \in E}\left(W C_{l}+R C_{l}\right) d_{l}$ Subject to:

$$
\sum_{k=1}^{K} Z_{k}^{m n}=1, \quad \forall(m, n) \in D
$$

$$
\begin{aligned}
& \sum_{w=1}^{W} X_{k i w}^{m n}=Z_{k}^{m n}, \\
& \forall(m, n) \in D, \forall k \in[1, K], \forall i \in\left[1, t_{k}^{m n}\right], \\
& \sum_{j=1}^{K^{\prime}} \sum_{w=1}^{W} Y_{l k j w}^{m n}=Z_{k}^{m n}, \\
& \forall(m, n) \in D, \forall k \in[1, K], \forall l \in P_{k}^{m n}, \\
& \sum_{(m, n) \in D} \sum_{k=1}^{K} \sum_{i=1}^{t_{k}^{m n}} X_{k i w}^{m n} \xi_{l k i}^{m n} \leq W C_{l}, \\
& \forall l \in E, \forall w \in[1, W], \\
& \sum_{(m, n) \in D} \sum_{k=1}^{K} \sum_{j=1}^{K^{\prime}} \beta_{\overline{l k}}^{m n} \gamma_{\overline{l k j l}}^{m n} Y_{l k j w}^{m n} \leq R C_{l}, \\
& \forall l, \bar{l} \in E, \bar{l} \neq l, \forall w \in[1, W] \\
& Z_{k}^{m n}, X_{k i w}^{m n}, Y_{l k j w}^{m n} \in\{0,1\}, \\
& W C_{l}, R C_{l} \in Z^{+} .
\end{aligned}
$$

The objective of this formulation is to minimize the cost of total fiber used. Constraint (13) ensures that among all working path candidates exactly one is chosen. In constraint (14), it is stated that for every section of a working path a wavelength should be assigned. This constraint allows that wavelength conversion can be performed at the endpoints of each section. Constraint (15) implies that for every link failure on a working path there is a restoration path assigned. Constraints (13-15) are the path and wavelength assignments for the formulation. Constraints (16-17) are link capacity assignments, that is, links are assigned sufficient working and restoration capacities for carrying all traffic demands and for achieving full restorability against all singlelink failures.

\subsubsection{Separate Design}

The formulation for separate design is the decoupled version of the joint formulation. Working and restoration path/capacity assignment problems are separated and solved sequentially. Furthermore, the restoration part of the problem is partitioned into multiple subproblems, that is, design of restoration capacities for each subnetwork is carried out separately.

Since this formulation is a partitioned version of the joint problem, the variables and indicator functions used in this formulation are the same as that of the joint one. In addition, we define the indicator function 
$\alpha_{l s}=1$, if link $l$ is in subnetwork $s$ in restoration layer. The working path/capacity assignment problem is discussed first, and the restoration part will be discussed subsequently.

Working Path and Capacity Assignment: The ILP formulation for the design of working capacities is given by

Objective: minimize $\sum_{l \in E} W C_{l} d_{l}$

Subject to:

$$
\begin{aligned}
& \sum_{k=1}^{K} Z_{k}^{m n}=1, \quad \forall(m, n) \in D, \\
& \sum_{w=1}^{W} X_{k i w}^{m n}=Z_{k}^{m n}, \\
& \forall(m, n) \in D, \forall k \in[1, K], \forall i \in\left[1, t_{k}^{m n}\right], \\
& \sum_{(m, n) \in D} \sum_{k=1}^{K} \sum_{i=1}^{t_{k}^{m n}} X_{k i w}^{m n} \xi_{l k i}^{m n} \leq W C_{l}, \\
& \forall l \in E, \forall w \in[1, W], \\
& Z_{k}^{m n}, X_{k i w}^{m n} \in\{0,1\}, \\
& W C_{l} \in Z^{+} .
\end{aligned}
$$

The objective of this formulation is to minimize the cost of total fiber used for working paths. Constraint (20) implies that only one working path is selected for each demand. Constraint (21) assigns a wavelength on each section of the selected working path. In constraint (22), the working capacity is determined such that all connections can be carried over the network.

Restoration Path and Capacity Assignment: Let $Q$ be the set of triplets $(m, n, k)$ defined as $Q=\left\{(m, n, k) \mid Z_{k}^{m n}=1\right\}$, where $\left\{Z_{k}^{m n}\right\}$ correspond to the values in the optimum solution of the ILP formulation for the design of working capacities given above. For each subnetwork $s$, let $E_{s}$ be the set of links belonging to subnetwork $s$ in the restoration layer, that is, RM for subnetwork $s$ is responsible for the restoration of failed connections when link $l \in E_{s}$ fails.

The ILP formulation for computing restoration paths and capacities given below is for subnetwork $s$. This problem is solved successively for each subnetwork.
Objective: minimize $\sum_{l \in E} R C_{l}^{s} d_{l}$ Subject to:

$$
\begin{aligned}
& \sum_{j=1}^{K^{\prime}} \sum_{w=1}^{W} Y_{l k j w}^{m n}=1, \\
& \quad \forall(m, n, k) \in Q, \forall l \in\left(E_{s} \cap P_{k}^{m n}\right), \\
& \sum_{(m, n, j) \in Q} \sum_{j=1}^{K^{\prime}} \beta_{\overline{l k}}^{m n} \gamma_{\overline{l k j l}}^{m n} \alpha_{\bar{l}} Y_{\overline{l k j w}}^{m n} \leq R C_{l}^{s}, \\
& \quad \forall \bar{l} \in L_{s}, \forall l \in E, \bar{l} \neq l, \forall w \in[1, W], \\
& Y_{l k j w}^{m n} \in\{0,1\}, \\
& R C_{l} \in Z^{+} .
\end{aligned}
$$

The objective of this formulation is to minimize the cost of total fiber used for restoration paths. Constraint (25) assigns a wavelength on one restoration path for each failed working path in case of failure of link $l \in E_{s}$. Constraint (26) determines the restoration capacity for each link in subnetwork $s$ in case of all possible single-link failures.

This formulation is implemented successively for all subnetworks. After all subnetworks are designed individually, restoration link capacity for a link is determined by taking the maximum over all assignments made by individual subnetworks sharing the link, that is,

$$
R C_{l}=\max _{s} R C_{l}^{s}
$$

\subsection{Numerical Results}

Joint and separate design methods are applied to 23node and 32-node mesh networks. For each network topology, four different sets of traffic demands are generated. We use the following parameters: number of candidate paths for working paths $K=K^{\prime}=4$, and number of wavelengths $W=8$. The ILP formulation was implemented on CPLEX 7.1.

Both partitionings obtained by the ILP formulation and the heuristic algorithm for the 23-node network were considered. For the joint design algorithm, the maximum running time of CPLEX was limited to 36 hours. For the 23-node network, the joint design produced suboptimum solutions within the run-time limit. For the 32-node network, the joint design was not able to generate a reasonable integer feasible solution within the run-time limit for all demand sets. With the separate design, the working part of the 
problem generated suboptimal solutions within 36 hours of running time, whereas the optimum solution was obtained for the restoration part.

In order to evaluate the performance of section restoration, we compare it with path rerouting. With path rerouting in all-optical networks, we assume that working and restoration paths are mutually linkdisjoint. For path restoration, there are two cases to be considered with respect to the locations of the transponders. First, in order to have a fair comparison with section restoration, we locate the transponders at the same locations as in section restoration. In this case, wavelength conversion can be exercised only at the boundaries of subnetworks. For the second case, in order to compute a lower bound on the fiber cost, we discuss path restoration with wavelength conversion at all nodes of the network. We call the former case as fair conversion and the latter case as the full conversion. In path restoration with fair and full conversion, network design problems are formulated as ILP problems, and joint and separate design methods are used for designing subnetworks as in the case of section restoration. Because of space limitations, these formulations are not presented here.

For the 23-node network, the four demand sets have $63,67,69$, and 63 demand pairs, respectively, and are randomly generated. With optimum ILP partitioning and parameters $R_{\max }=2500 \mathrm{~km}, L_{\max }=24$ and $N_{\text {max }}=16$, the fiber costs for section restoration and path restoration/fair conversion and path restoration/ full conversion are shown for joint and separate designs in Table 1.

We observe from this table that with all three restoration techniques there are cases where separate design generates lower fiber cost than the joint design. This is due to the fact that the results marked with " **, are suboptimal as obtained within the 36-hour

Table 1. Total fiber costs for the 23-node network partitioned by ILP formulation with $R_{\max }=2500 \mathrm{~km}, N_{\max }=16 \quad$ and $L_{\max }=24\left(^{*}\right.$ : suboptimal $)$.

\begin{tabular}{|c|c|c|c|c|c|c|}
\hline \multirow{2}{*}{$\begin{array}{l}\text { Demand } \\
\text { Set }\end{array}$} & \multicolumn{2}{|c|}{ Section } & \multicolumn{2}{|c|}{ Path (Fair) } & \multicolumn{2}{|c|}{ Path (Full) } \\
\hline & Joint & Separate & Joint & Separate & Joint & Separate \\
\hline 1 & $24136^{*}$ & 22124 & 22275 & 19761 & 19327 & 19641 \\
\hline 2 & 24838 & 25347 & $26396^{*}$ & 22478 & $22821 *$ & 22478 \\
\hline 3 & 22715 & 24280 & $23934 *$ & 22057 & 21361 & 21784 \\
\hline 4 & 21581 & 23866 & 21095 & 21580 & 20391 & 20771 \\
\hline
\end{tabular}

runtime limit. Decoupled subnetwork design problems in separate design have lower computational complexity, and they may generate better results within the time limits. In general, for all restoration methods the costs for the joint and separate designs are close. An important observation is that the largest difference between fiber costs obtained with section restoration/separate design and path restoration/fair conversion is $13.1 \%$. The above difference increases to $17.0 \%$ if we allow full conversion in path restoration.

The above analysis is repeated for the 23-node network partitioned with the heuristic algorithm. The results are shown in Table 2 . These results present similar characteristics with those for the ILP partitioned network. Since in path restoration/full conversion the network cost is independent of partitioning, the same fiber costs are obtained in that case as those in Table 1. With this partitioning, the largest difference between fiber costs obtained with section restoration/separate design and path restoration/fair conversion is $3.0 \%$. The above difference increases to $9.5 \%$ if we allow full conversion in path restoration. Both numbers are smaller than those obtained with the ILP partitioned network.

We repeat the above experiments for the 32-node network which is partitioned by the heuristic algorithm with parameters $R_{\max }=3000 \mathrm{~km}$, $L_{\max }=24$, and $N_{\max }=16$. Four demand sets with 89, 73, 86, and 66 demand pairs, respectively, are randomly generated. Fiber costs for section restoration/separate design, path restoration/fair conversion/ separate design, and path restoration/full conversion for joint and separate designs are given in Table 3.

Fiber costs of section restoration/separate design, and path restoration/fair conversion/separate design are close to each other, and the highest difference is

Table 2. Total fiber costs for the 23-node network partitioned by the heuristic algorithm with $R_{\max }=2500 \mathrm{~km}, N_{\max }=16$ and $L_{\max }=24\left(^{*}\right.$ : suboptimal $)$.

\begin{tabular}{|c|c|c|c|c|c|c|}
\hline \multirow{2}{*}{$\begin{array}{l}\text { Demand } \\
\text { Set }\end{array}$} & \multicolumn{2}{|c|}{ Section } & \multicolumn{2}{|c|}{ Path (Fair) } & \multicolumn{2}{|c|}{ Path (Full) } \\
\hline & Joint & Separate & Joint & Separate & Joint & Separate \\
\hline 1 & $20944^{*}$ & 20340 & $19865^{*}$ & 19764 & 19327 & 19641 \\
\hline 2 & $24183^{*}$ & 24125 & $25979 *$ & 23821 & $22821^{*}$ & 22478 \\
\hline 3 & $23174^{*}$ & 23381 & $23858^{*}$ & $23585^{*}$ & 21361 & 21784 \\
\hline 4 & $21453^{*}$ & 20440 & $21432 *$ & $20825^{*}$ & 20391 & 20771 \\
\hline
\end{tabular}


Table 3. Total fiber costs for the 32-node network partitioned by the heuristic algorithm with $R_{\max }=3000 \mathrm{~km}, N_{\max }=16$ and $L_{\max }=24\left(^{*}\right.$ : suboptimal).

\begin{tabular}{|c|c|c|c|c|}
\hline \multirow{2}{*}{$\begin{array}{l}\text { Demand } \\
\text { Set }\end{array}$} & \multirow{2}{*}{$\frac{\text { Section }}{\text { Separate }}$} & \multirow{2}{*}{$\frac{\text { Path (Fair) }}{\text { Separate }}$} & \multicolumn{2}{|c|}{ Path (Full) } \\
\hline & & & Joint & Separate \\
\hline 1 & 45212 & 42129 & $44452^{*}$ & 42107 \\
\hline 2 & 40360 & 39303 & $39230 *$ & 36903 \\
\hline 3 & 44151 & 43856 & $41957 *$ & 41974 \\
\hline 4 & 36221 & $37416^{*}$ & $35724 *$ & 35256 \\
\hline
\end{tabular}

Table 4. Total number of transponders for translucent and opaque networks with the 32-node network partitioned by the heuristic algorithm with $R_{\max }=3000 \mathrm{~km}, N_{\max }=16$ and $L_{\max }=24$.

\begin{tabular}{lll}
\hline Demand Set & Translucent & Opaque \\
\hline 1 & 320 & 1830 \\
2 & 295 & 1670 \\
3 & 312 & 1828 \\
4 & 280 & 1632 \\
\hline
\end{tabular}

7.3\%. This difference remains unchanged when full wavelength conversion is allowed for path restoration.

The number of transponders used by translucent networks with section section restoration and opaque networks are shown in Table 4 for the 32-node network with the network partitioning shown in Fig. 5. The results show that the transponder cost is substantially reduced for the translucent network compared to the opaque network.

\section{Conclusions}

The network partitioning problem in translucent optical networks has been studied as an optimization problem, and an ILP formulation for the problem has been presented. Due to the high computational complexity of the ILP optimization, we presented an alternative greedy heuristic algorithm for planar networks that performs comparable with the ILP formulation in terms of the number of subnetworks in the partitioning.

Section restoration with separate design is computationally more tractable since several smaller sized problems are solved separately instead of solving a singe large sized problem. Section restoration also generates fiber costs that are competitive with theoretically more efficient path restoration.
Numerical results demonstrate that the section restoration with separate design produces fiber costs that are generally within $10 \%$ of those with path restoration/joint design when fair conversion is used. Moreover, fiber costs with section restoration are also within $15 \%$ of those with path restoration/full conversion. It is also observed that fiber costs with section restoration with separate design are 4-16\% lower when the network partitioned with the heuristic algorithm is used.

It has also been shown that the transponder cost is substantially reduced in translucent optical networks compared to opaque networks. Coupled with the relatively small increase in fiber costs, this huge reduction in transponder costs make translucent optical networks a viable architecture for future optical networks.

\section{Acknowledgment}

Work partially supported by the Scientific and Technological Research Council of Turkey (TUBITAK) under the project 199E005.

\section{References}

[1] S. B. Alexander, et al., A precompetitive consortium on wideband all-optical networks, IEEE/OSA Journal of Lightwave Technology, vol. 11, no. 5/6, (May/June 1993), pp. 714-735.

[2] R. E. Wagner, et al., MONET: Multiwavelength optical networking, IEEE/OSA Journal of Lightwave Technology, vol. 14, no. 6, (June 1996), pp. 1349-1355.

[3] J. M. H. Elmirghani, H. T. Mouftah, All-optical wavelength conversion: Technologies and applications in DWDM networks, IEEE Communications Magazine, vol. 38, no. 3, (March 2000), pp. 86-92.

[4] E. Karasan, E. Ayanoglu, Performance of WDM transport networks, IEEE Journal on Selected Areas in Communications, vol. 16, no. 7, (Sept. 1998), pp. 1081-1096.

[5] J. M. Yates, M. P. Rumsewicz, J. P. R. Lacey, Wavelength converters in dynamically-reconfigurable WDM networks, IEEE Communication Surveys, Second Quarter (1999), pp. 215.

[6] B. Ramamurthy, B. Mukherjee, Wavelength conversion in WDM networking, IEEE Journal on Selected Areas in Communications, vol. 16, no. 7, (Sept. 1998), pp. 10611073.

[7] K. Bala, R. Cordell, E. Goldstein, The case for opaque multiwavelength lightwave networks, Proceedings of IEEE/ LEOS Summer Topical Meeting on Global Information Infrastructure, (Keystone, CO, Aug. 1995). 
[8] P. Green, Progress in optical networking, IEEE Communications Magazine, vol. 39, no. 1, (Jan. 2001), pp. 54-61.

[9] B. Ramamurthy, et al., Transparent vs. opaque vs. translucent wavelength-routed optical networks, Technical Digest, Optical Fiber Communication (OFC'99), (San Diego, CA, Feb. 1999), pp. 59-61.

[10] B. Rajagopalan, et al., IP over optical networks: Architectural aspects, IEEE Communications Magazine, vol. 38, no. 9, (Sept. 2000), pp. 94-102.

[11] J. Strand, A. L. Chiu, R. Tkach, Issues for routing in the optical layer, IEEE Communications Magazine, vol. 39, no. 2, (Feb. 2001), pp. 81-87.

[12] E. Yetginer, E. Karasan, Regenerator placement and traffic engineering with restoration in GMPLS networks, Photonic Network Communications, vol. 6, no. 2, (Sept. 2003), pp. 139-149.

[13] C. Ou, H. Zang, B. Mukherjee, Sub-path protection for scalability and fast recovery in WDM mesh networks, Technical Digest, Optical Fiber Communication (OFC'2002), (Anaheim, CA, March 2002), pp. 495-496.

[14] P.-H. Ho, H. Mouftah, A framework for service-guaranteed shared protection in WDM mesh networks, IEEE Communications Magazine, vol. 40, no. 2, (Feb. 2002), pp. $97-103$.

[15] M. Alanyali, E. Ayanoglu, Provisioning algorithms for WDM optical networks, IEEE/ACM Transactions on Networking, vol. 7, no. 5, (Oct. 1999), pp. 767-778.

[16] G. Mohan, C. S. R. Murthy, Lightpath restoration in WDM optical networks, IEEE Network, vol. 14, (Nov./Dec. 2000), pp. 24-32.

[17] R. R. Iraschko, W. D. Grover, A highly efficient pathrestoration protocol for management of optical network transport integrity, IEEE Journal on Selected Areas in Communications, vol. 18, no. 5, (May 2000), pp. 779-794.

[18] B. T. Doshi, et al., Optical network design and restoration, Bell Labs Technical Journal, vol. 4, no. 1, (Jan.-March 1999), pp. 58-84.

[19] R. K. Ahuja, T. L. Magnanti, J. B. Orlin, Network Flows (Prentice Hall, New Jersey, 1993).
[20] Béla Bollobás, Graph Theory (Springer-Verlag, New York, 1979).

[21] T. Nishizeki, N. Chiba, Planar Graphs: Theory and Algorithms (North Holland, 1988).

Ezhan Karasan received the B.S degree from Middle East Technical University, Ankara, Turkey, the M.S. degree from Bilkent University, Ankara, Turkey, and the Ph.D. degree from Rutgers University, Piscataway, New Jersey, all in electrical engineering, in 1987, 1990, and 1995 , respectively.

During 1995-1996, he was a postdoctorate researcher at Bell Labs, Holmdel, New Jersey. From 1996 to 1998, he was a Senior Technical Staff Member in the Lightwave Networks Research Department at AT\&T Labs-Research, Red Bank, New Jersey. Since 1998, he has been an assistant professor in the Electrical and Electronics Engineering Department at Bilkent University, Ankara, Turkey.

His current research interests are in the application of optimization and performance analysis tools for the design and analysis of optical and wireless ad hoc networks.

Mustafa Arisoylu received the B.S. and M.S. degrees in electrical and electronics engineering from Bilkent University, Ankara, Turkey in 1999 and in 2001, respectively. He is currently studying in the Ph.D. program in electrical and computer engineering at University of California San Diego.

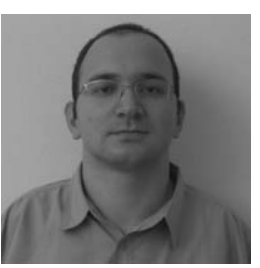

